cohorts of contemporary workers. 169 work-related exposures had been investigated in relation to retirement outcomes. The exposures were grouped into 19 categories to enable comparison and synthesis. The included studies were heterogenous in terms of outcome definitions and measurement of exposures. However, appreciation at work and higher job control consistently associated with a decreased risk of retirement. The review also highlighted limited evidence that: age discrimination: having a positive culture of working beyond SPA: flexible working hours: and job prospects may influence retirement.

Conclusion Increasing worker's job control and perception of appreciation at work have may delay retirement decisions. Further research is required to explore the effect of workrelated factors on retirement in cohorts of contemporary workers.

\section{P-206 'ASSESSING THE IMPACT OF EXPOSURE CONTROL ON FUTURE CANCER BURDEN AMONG CONSTRUCTION WORKERS'}

${ }^{1}$ Chaojie Song, Kate Jardine, Victoria H Arrandale, Amir Mofidi, Emile Tompa, Thomas Tenkate, Hugh Davies, Paul Demers. 'Ontario Health, Canada

\subsection{6/OEM-2021-EPI.234}

Objectives Construction workers are exposed to several carcinogens at work. Implementing intervention methods may reduce workers' exposure, which should subsequently reduce the number of cancer cases attributable to the exposure. The current study estimates the future cancer burden due to several common carcinogens among Ontario construction workers, and assesses the impact of implementing interventions on this burden. This presentation focuses on solar ultraviolet radiation and asbestos.

Methods The annual number of new cancer cases attributable to each carcinogen was estimated from 2030 to 2060 using Levin's equation based on the prevalence of exposure (PrE) and the risk of cancer (RR) associated with exposure. The RR was selected from a review of the epidemiologic literature. The PrE was estimated using CAREX Canada's estimates of prevalence and level of exposure, combined with historical and projected employment data, labour force characteristics, and survival probabilities. The intervention methods specific to each carcinogen were assumed to be fully implemented from 2020, and incorporated into the model by adjusting prevalence and level of exposure downwards.

Results We estimated that without intervention, 27645 nonmelanoma skin cancers would be attributable to sun exposure in Ontario construction workers from 2030 to 2060. Using portable shade structure and hat/long sleeve clothes, a total of 1957 and 2503 of these cases would be prevented, respectively. For asbestos, the two interventions, asbestos ban and building registry, would prevent 56 and 439 lung cancers out of the 6022 attributable cases from 2030 to 2060 if no intervention was applied.

Conclusions Future work-related cancers can be prevented by reducing workers' exposure. Combining the economic assessment of both the cancer burden and the costs of implementing exposure controls will help to assess the cost-benefit of different intervention methods, which can be used to direct intervention strategies in construction workplace.

\section{P-212 'RETURN TO WORK AFTER VOCATIONAL REHABILITATION'}

${ }^{1}$ Bianca Biason Albuquerque, Vivian Mininel. 'Universidade Federal de São Carlos, Brazil

\subsection{6/OEM-2021-EPI.235}

Introduction Changes in the workplace environment, precarization and flexibilization of employment, loss of labor rights, and the big economy have had an impact, increasing work accidents, harms, and occupational diseases as well sick leaves. In Brazil, Vocational Rehabilitation (VR) is provided by the National Institute of Social Security system and includes educational, adjustment, and re-adjustment support to facilitate return to work among workers who receive benefits due disease or accidents. However, its scope is quite limited and does not offer a follow-up to workers after discharge.

Objective To understand the return to work after discharge of the VR.

Methods Qualitative study developed in a VR program from Sao Paulo state and carried out in two phases: (i) documental analysis to identify potential participants who were assisted by VR; (ii) individual interviews by telephone following an open questions script and analyzed through Thematic Content Analysis. This research was approved by the Ethical Committee of Federal University of Sao Carlos; all ethical concerns were addressed.

Results From 2009 to 2020, 263 workers returned to work after discharge of VR: 84 (32\%) women and 179 (68\%) men received support predominantly due to musculoskeletal disorders (35\%) and external causes of injuries (33\%). Of these, $71(27 \%)$ returned to the same task and $192(73 \%)$ to different ones. 33 workers discharged from VR during 2019-2020 were invited to participate in the interviews and 11 workers were interviewed. Three categories emerged from interviews: difficulties in the return to work process; limits of VR to return to work; necessary advances to VR regard to return to work.

Conclusion Results showed the need for reorganization of VR including review of criteria of inclusion, interprofessional team analysis of potential participants, discharge mechanisms, follow-up of the worker after discharge, and negotiating with employers accommodation to the rehabilitated worker.

\section{P-213 'USING PARITY MONITOR AND RANDOM FOREST MODELS TO EXPLORE INDICATORS OF INDOOR AIR QUALITY FOR INDEPENDENT WORKERS IN COFFEE SHOPS'}

${ }^{1}$ HsiChen Liu, Chin-Sheng Tang, Tzu-Ying Lee. 'Chinese Culture University, Taiwan

\subsection{6/OEM-2021-EPI.236}

Introduction Independent workers often work in Taiwan's coffee shops. However, there is not enough information for workers to notice poor indoor air quality (IAQ), such as high concentration of particulate matters (PMs) and carbon dioxide (CO2).

Objectives This study aims to explore indicators of IAQ for independent workers in the coffee shop industry. 